\title{
Partial jamming and non-locality in dense granular flows
}

\author{
Prashidha Kharel ${ }^{1, \star}$ and Pierre Rognon ${ }^{1, \star \star}$ \\ ${ }^{1}$ Particles and Grains Laboratory, School of Civil Engineering, \\ The University of Sydney, 2006 NSW, Australia
}

\begin{abstract}
Dense granular flows can exhibit non-local flow behaviours that cannot be predicted by local constitutive laws alone. Such behaviour is accompanied by the existence of diverging cooperativity length. Here we show that this length can be attributed to the development of transient clusters of jammed particles within the flow. By performing DEM simulation of dense granular flows, we directly measure the size of such clusters which scales with the inertial number with a power law. We then derive a general non-local relation based on kinematic compatibility for the existence of clusters in an arbitrary non-homogenous flow. The kinematic nature of this derivation suggests that non-locality should be expected in any material regardless of their local constitutive law, as long as transient clusters exist within the flow.
\end{abstract}

\section{Introduction}

The bulk rheology of dense granular flows can be described by a local constitutive law involving an effective viscosity and yield stress, both depending on the normal stress [1-3]. However, this local description fails to capture the behaviour of flows in heterogeneous configurations, in particular, flows near walls or when stress and shear strain-rate gradients are present in the flow. In such configurations, the influence on the flow at one point due to flows at its neighbouring points results in nontrivial flow features, e.g., S-shaped velocity profile in plane shear flow between two walls $[4,5]$ and wide shear bands observed in slit-bottom cell experiments [6].

Various non-local models have been introduced that successfully account for these situations [4, 5, 7-12]. In spite of the differences in their formulations, these nonlocal models are all based on the key assumption that there exists a meso-scale length $\ell$ which is larger than the grain size $d$ and that it represents the extent of non-locality, i.e., the influence on the flow at one point due to flows at its neighbouring points. An association of this length with the critical stopping height of grains on inclined plane [1315] has been made [16], which suggested possible link between jamming and non-locality. Here we show how this length representing non-locality can be attributed to the development of correlated structures of jammed particles within the flow.

\section{Multiple shear bands and clusters in homogeneous shear flow}

In order to study the microstructure of the dense granular flows, we perform discrete element simulation, as in [5], of a two dimensional assembly of 10,000 grains sheared homogeneously with a shear strain-rate of $\dot{\gamma}$ under constant normal pressure $P$, as shown in Fig. 1 . The grains have density $\rho$, mean diameter $d$ and a polydispersity of $\pm 20 \%$ in order to avoid crystallisation and any permanent shear banding. Bi-periodic Lees-Edwards boundary condition [17] is used to ensure the time averaged shear flow is homogeneous. Overlapping grains interact via normal inelastic forces and tangential frictional forces with Young's modulus $E$, coefficient of restitution $e=0.5$, and grain-tograin coefficient of friction $\mu_{g}=0.5$. The typical overlap is of the order of $\kappa=P / E=10^{-3}$. The system is initially set randomly with no contacts, then the pressure $P$ and the shear strain rate $\dot{\gamma}$ are applied. All measurements reported here are after the system has reached a steady state.

As a way to identify correlated structures, triplets of neighbouring grains are identified using a Delaunay triangulation and a criteria is employed to determine which triplets are jammed. Amongst several possibilities, we used a criteria based on the maximum normal relative velocity between pair of grains in the triplet $\mathrm{k}:|\Delta v|=$ $\max \left(\left|\Delta v_{12}^{k}\right| ;\left|\Delta v_{13}^{k}\right| ;\left|\Delta v_{23}^{k}\right|\right)$ (see Fig. 1(a)). The triplet is considered kinematically jammed if all of the three relative normal velocities are smaller than the average relative normal velocity $\langle|\Delta v|\rangle$ between all pair of neighbouring grains in the full system. The flow is composed of transient orthogonal shear bands that create a lattice of highly sheared zones $[18,19]$ delimiting the boundaries of cluster of grains subjected to little if any shear deformation, thus moving briefly like rigid bodies. We then count the num-

\footnotetext{
${ }^{\star}$ e-mail: prashidha.kharel@sydney.edu.au
}

$\star \star$ e-mail: pierre.rognon@sydney.edu.au 


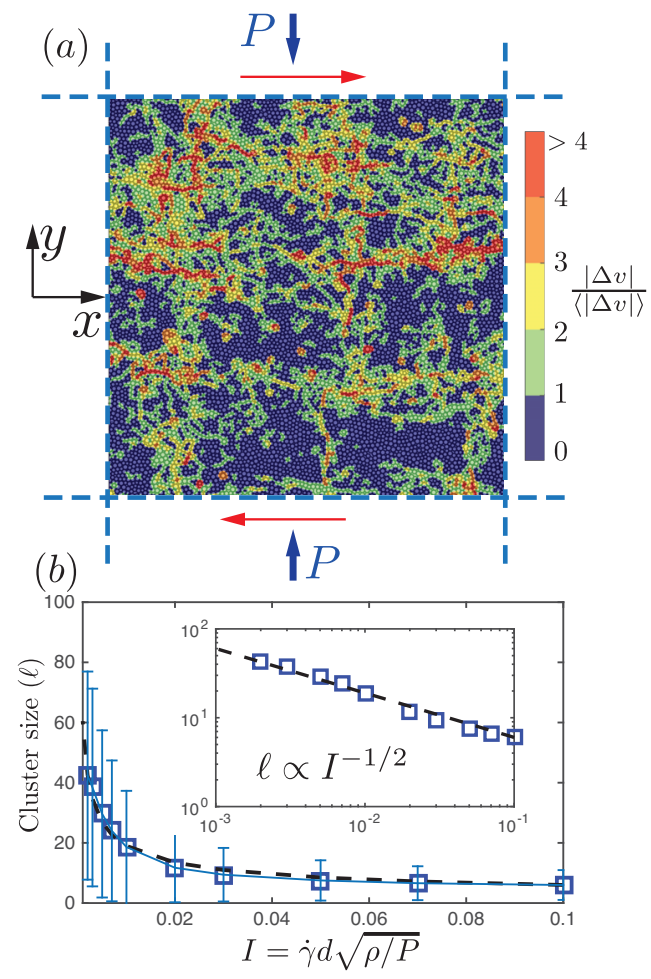

Figure 1. Clusters in sheared granular flows: (a) System of 10000 grains sheared homogeneously by using bi-periodic boundary condition. The colours represent a snapshot of relative velocities between the grains for inertial number $I=0.001$. The partially jammed clusters are shown in dark blue (b) The average cluster size (squares) and standard deviation (vertical bars) for different inertial numbers. Inset: same plot in log-log axis. Dashed curves indicate a power law $-1 / 2$.

ber of grains, $n$, in each clusters and calculate its size as $\ell=\sqrt{n} d$. The average size of such jammed clusters scale with the inertial number with the following power law, as shown in Fig 1 (b):

$$
\ell \propto \frac{d}{\sqrt{I}}
$$

Similar scaling has been observed for other non-local length scales $[5,10,11]$ measured indirectly in various systems, which suggests a possible relation between the clusters and non-locality.

\section{Derivation of continuum non-local relation}

Let us now seek to derive a general relation for non-local rheology by considering the existence of correlated structures in an arbitrary non-homogeneous flow. The first assumption is that, in the dense regime, the flow is comprised of transient clusters of grains that move like rigid bodies. Since, rigid body motion will result in constant vorticity throughout the cluster size, such clusters will redistribute the vorticity over their size. The local value of the vorticity within a flow should therefore be affected by the value of the vorticity in the surrounding.

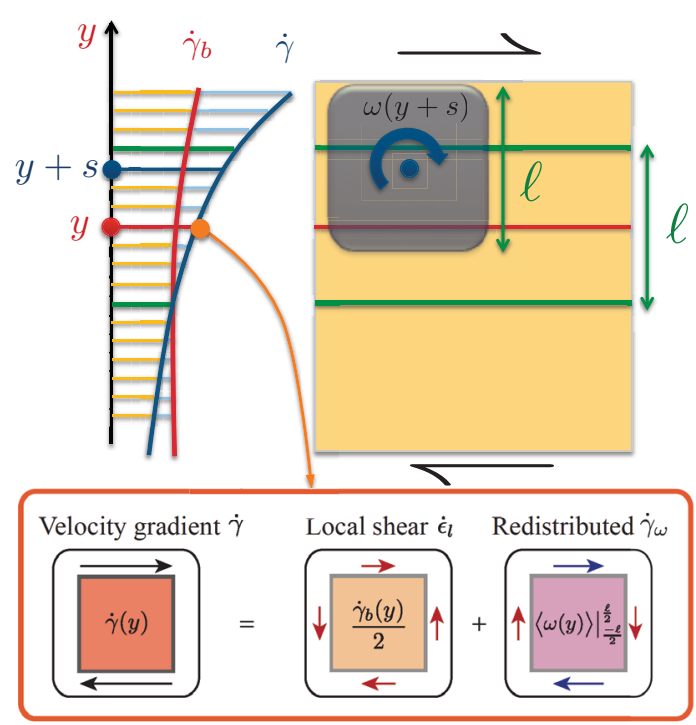

Figure 2. Illustration of the proposed mechanism at the origin of non-locallity. (Top) Actual shear rate profile $\dot{\gamma}(y)$ in a heterogeneous sheared layer compared to the shear rate profile $\dot{\gamma}_{b}$ predicted by a local constitutive law with no account for nonlocal effects; A clusters of jammed particles located at a position $y+s$ is represented, distributing its vorticity over its size $\ell$. (bottom) The local velocity gradient is comprised of i) a local pure shear strain rate $\dot{\epsilon}=\dot{\gamma}_{b} / 2$ governed by the local stresses via a local constitutive law, and a contribution $\dot{\gamma}_{\omega}=\left.\langle\omega(y)\rangle\right|_{-\ell / 2} ^{\ell / 2}$ coming from the vorticities of nearby clusters.

The second assumption is that the velocity gradient can be decomposed into a local shear strain deformation that is not redistributed, $\dot{\epsilon}_{l}$, and a shear rate due to redistribution of vorticity, $\dot{\gamma}_{\omega}$ :

$$
\dot{\gamma}(y)=\dot{\epsilon}_{l}(y)+\dot{\gamma}_{\omega}(y)
$$

The local shear deformation is responsible for mechanical dissipation in localised shear bands, which shall follow the local constitutive law. On the other hand, the vorticity governs the cluster rotation rate which gets redistributed along the transverse direction.

As shown in figure 2, when a cluster of size $\ell$ develops at some position $y+s$, it redistributes the vorticity $\omega(y+s)=\dot{\gamma}(y+s) / 2$ over the zone comprised over its size $\ell$. Given that these clusters are transient and develop at different locations, the net shear rate at a point $y$ due to redistribution of vorticity is given by the average of the vorticities of points between $y \pm \ell / 2$, i.e.,

$$
\dot{\gamma}_{\omega}(y)=\left.\langle\omega(y)\rangle\right|_{-\ell / 2} ^{\ell / 2}=\frac{1}{\ell} \int_{-\ell / 2}^{\ell / 2}(\dot{\gamma}(y+s) / 2) d s
$$

By contrast, local pure shear is not redistributed, which is entirely governed by the local stresses in the layer and is therefore the same as in the case of homogeneous shear flow, i.e., $\dot{\epsilon}_{l}(y)=\dot{\epsilon}_{b}(y)=\dot{\gamma}_{b}(y) / 2$, where $\dot{\gamma}_{b}$ is the shear rate predicted by the local constitutive law. Substituting these two results in (2) leads to: 


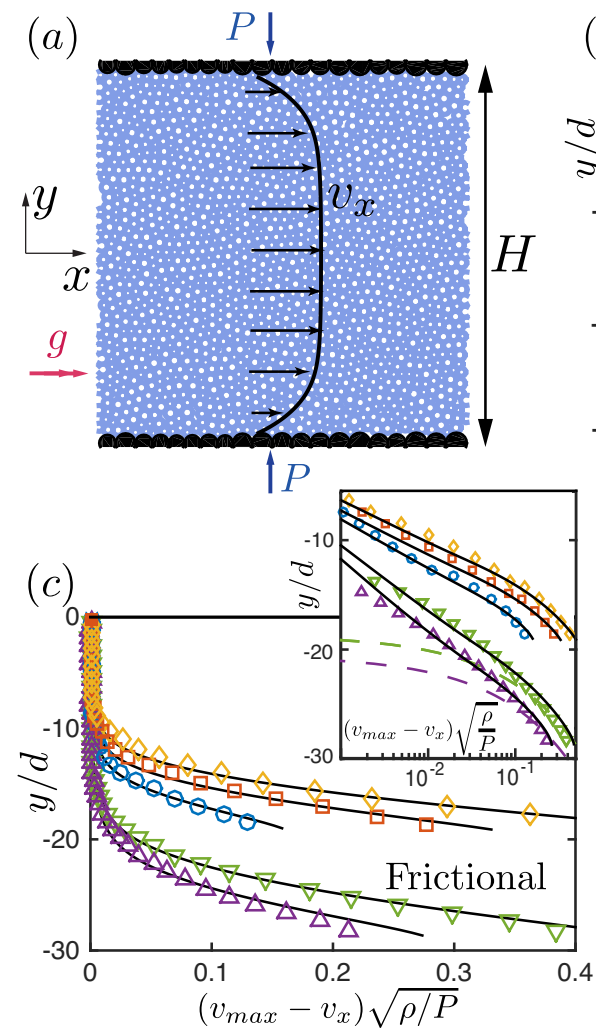

$(b)$

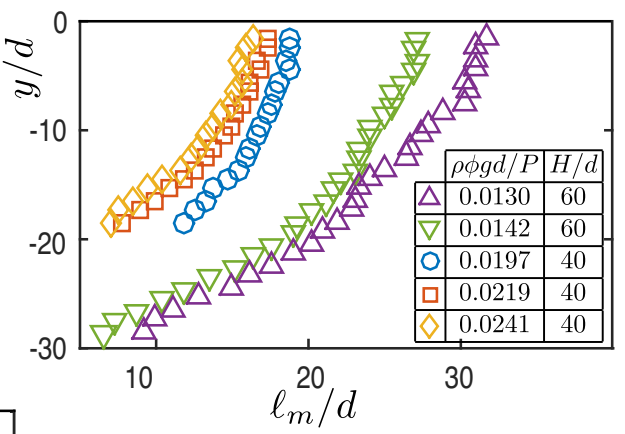

$(d)$

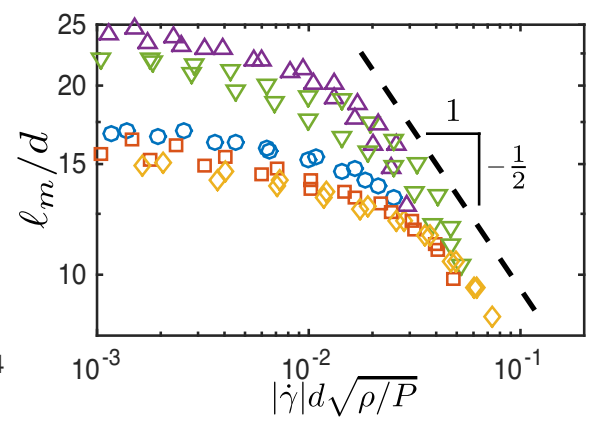

Figure 3. Non-locality and clusters in a non-homogeneous shear flow. (a) $H \times H$ Poiseuille flow geometry where wall grains (black) with average diameter $2 d$ are stationary along $x$ and subjected to a pressure $P$ along $y$, whereas flowing grains (blue) with average diameter $d$ are subjected to a constant force of $\pi d^{2} \rho g / 4$ along $x$. Periodic boundary condition is used along the $\mathrm{x}$ axis. (b) Measured cluster size profile, (c) velocity profile [inset: same in semi-log scale], and (d) measured cluster size versus inertial number within various Poiseuille flows. Symbols represent data from DEM simulations, solid black curves in (c) are solutions of eq. (4) and dashed lines in the inset of (c) are velocity predictions using local constitutive law for $\mathrm{H} / \mathrm{d}=60$.

$$
2 \dot{\gamma}(y)=\dot{\gamma}_{b}(y)+\frac{1}{\ell} \int_{-\ell / 2}^{\ell / 2} \dot{\gamma}(y+s) d s
$$

By using a Taylor expansion of $\dot{\gamma}(y+s)$ with respect to $s$ about $s=0$, the integral in (3) becomes:

$$
\begin{gathered}
\frac{1}{\ell} \int_{-\ell / 2}^{\ell / 2}\left(\dot{\gamma}(y)+\frac{\partial \dot{\gamma}(y)}{\partial y} s+\frac{1}{2} \frac{\partial^{2} \dot{\gamma}(y)}{\partial y^{2}} s^{2}+O\left(\frac{\partial^{3} \dot{\gamma}}{\partial y^{3}}\right)\right) d s \\
=\dot{\gamma}(y)+\frac{\ell^{2}}{24} \frac{\partial^{2} \dot{\gamma}(y)}{\partial y^{2}}+O\left(\frac{\partial^{4} \dot{\gamma}}{\partial y^{4}}\right)
\end{gathered}
$$

Introducing the second order approximation of this expression into (3) leads to a non-local equation governing the shear rate $\dot{\gamma}(y)$ :

$$
\dot{\gamma}(y)-\dot{\gamma}_{b}(y)=\frac{\ell^{2}}{24} \frac{\partial^{2} \dot{\gamma}}{\partial y^{2}} .
$$

This equation was derived solely based on kinematic compatibility, so it should be valid for any material regardless of their local constitutive law, as long as transient clusters of size $\ell$ exists in the flow. This differential equation is similar to the relation used in the Kinetic Elastoplastic (KEP) model [8-10], except that, here, the fluidity is replaced by shear strain-rate. In the quasi-static limit, the KEP model reduces to (4).

\section{Non-locality in non-homogeneous shear flow}

In order to assess the validity of the non-local model (4), we now consider a series of granular flows in a Poiseuille geometry (see Fig. 3(a)). This geometry involves a stress gradient, expressed as $\mu(y)=\tau(y) / P=\frac{\rho \phi g}{P} y$ for a solid fraction $\phi$, and the presence of walls, which both induce non-local effects [5, 10]. Figure 3(b) shows the profile of cluster size $\ell_{m}(y)$, which is measured using a protocol similar to that in the homogeneous case, measuring the average relative velocity for each horizontal layer across the flow. Figure 3(c) shows that the model (4) is able to reproduce all the measured velocity profiles. To calculate the bulk shear rate $\dot{\gamma}_{b}$, we use the constitutive relation of the form $\mu=\mu_{o}+b I$, where $\mu_{o}=0.266, b=1.54$, fitted from the plane shear flow data. For the cluster size we used $\ell(y)=A \ell_{m}(y)$, where $A=0.55$ is the single fitting constant which depends on the protocol used to measure the cluster size ${ }^{1}$. The two boundary conditions used are the values of the shear rate at both walls directly measured from the DEM simulations. Using Neumann boundary condi-

\footnotetext{
${ }^{1}$ In order to be consistent, we have used instantaneous velocities to measure cluster size. Using time averaged velocities does affect the absolute values of measured cluster size. However, the relative power law scaling in (1) is not affected.
} 
tions, as in [10, 12], also produces similar result. Figure 3(d) shows that size of clusters and local inertial numbers follow a power law consistent with (1) for smaller cluster sizes. However, larger cluster sizes seemingly saturate to some finite size, which may be attributed to a finite size effect of the system [5].

\section{Conclusions}

Here we showed that in the dense regime, granular flow is composed of the correlated structures of jammed particles. The typical size of such correlated structure scales with the inertial number with a power law, which is consistent with the scaling of non-local length measured in various systems $[5,10,11]$. By considering the existence of such structures in an arbitrary non-homogeneous flows, we derived a general non-local relation which is similar in form to the KEP model. The fact that this relation was derived solely based on kinematics of the flow suggests that it should be valid for any material regardless of their constitutive law, as long as transient clusters exists in the flow.

More generally, correlated motion of particles [2022], and avalanche-like rearrangements have been observed in various glassy materials like sand [23], granular materials [15, 24, 25], cohesive grains [26], foams [27], suspensions [8, 28], colloids [29, 30], and Lennard-Jones glass $[31,32]$. These observations suggest that clusters of particles may exist in many glassy materials. Our derivation indicates that non-local behaviours should then be expected. Furthermore, the link made here between clusters and non-locality creates a promising opportunity to better interpret, analyse and unify the non-local behaviours of glassy materials through a thorough characterisation of cluster size and formation mechanisms for specific materials and flow configurations.

\section{References}

[1] G. MiDi, The European Physical Journal E 14, 341 (2004)

[2] F. da Cruz, S. Emam, M. Prochnow, J.N. Roux, F. Chevoir, Physical Review E 72, 021309 (2005)

[3] P. Jop, Y. Forterre, O. Pouliquen, Nature 441, 727 (2006)

[4] T. Miller, P. Rognon, B. Metzger, I. Einav, Physical review letters 111, 058002 (2013)

[5] P.G. Rognon, T. Miller, B. Metzger, I. Einav, Journal of Fluid Mechanics 764, 171 (2015)

[6] D. Fenistein, M. van Hecke, Nature 425, 256 (2003)

[7] O. Pouliquen, Y. Forterre, Philosophical Transactions of the Royal Society A: Mathematical, Physical and Engineering Sciences 367, 5091 (2009)
[8] J. Goyon, A. Colin, G. Ovarlez, A. Ajdari, L. Bocquet, Nature 454, 84 (2008)

[9] L. Bocquet, A. Colin, A. Ajdari, Physical review letters 103, 036001 (2009)

[10] K. Kamrin, G. Koval, Physical Review Letters 108, 178301 (2012)

[11] M. Bouzid, M. Trulsson, P. Claudin, E. Clément, B. Andreotti, Physical review letters 111, 238301 (2013)

[12] D.L. Henann, K. Kamrin, Proceedings of the National Academy of Sciences 110, 6730 (2013)

[13] A. Daerr, S. Douady, Nature 399, 241 (1999)

[14] D. Ertaş, T.C. Halsey, EPL (Europhysics Letters) 60, 931 (2002)

[15] O. Pouliquen, Physical Review Letters 93 (2004)

[16] K. Kamrin, D.L. Henann, Soft Matter 11, 179 (2015)

[17] A. Lees, S. Edwards, Journal of Physics C: Solid State Physics 5, 1921 (1972)

[18] M. Falk, J. Langer, Physical Review E 57, 7192 (1998)

[19] M.L. Falk, J. Langer, Annual Review of Condensed Matter Physics 2, 353 (2011)

[20] R. Khosropour, J. Zirinsky, H.K. Pak, R.P. Behringer, Physical Review E 56, 4467 (1997)

[21] F. Radjai, S. Roux, Physical review letters 89, 064302 (2002)

[22] P. Rognon, I. Einav, Physical Review Letters 105 (2010)

[23] S. Abedi, A.L. Rechenmacher, A.D. Orlando, Granular Matter 14, 695 (2012)

[24] A.S. Keys, A.R. Abate, S.C. Glotzer, D.J. Durian, Nature Physics 3, 260 (2007)

[25] S. Henkes, D.A. Quint, Y. Fily, J. Schwarz, Physical review letters 116, 028301 (2016)

[26] P.G. Rognon, J.N. Roux, M. Naaim, F. Chevoir, Journal of Fluid Mechanics 596, 21 (2008)

[27] D. Durian, Physical Review E 55, 1739 (1997)

[28] G. Düring, E. Lerner, M. Wyart, Physical Review E 89 (2014)

[29] L. Berthier, G. Biroli, J.P. Bouchaud, L. Cipelletti, D.E. Masri, D. L'Hôte, F. Ladieu, M. Pierno, Science 310, 1797 (2005)

[30] P. Ballesta, A. Duri, L. Cipelletti, Nature Physics 4, 550 (2008)

[31] C. Donati, S.C. Glotzer, P.H. Poole, W. Kob, S.J. Plimpton, Phys. Rev. E 60, 3107 (1999)

[32] A. Lemaître, C. Caroli, Physical review letters 103, 065501 (2009) 